Article

\title{
Constructions of Helicoidal Surfaces in a 3-Dimensional Complete Manifold with Density
}

\author{
Önder Gökmen Yıldız \\ Department of Mathematics, Faculty of Arts and Sciences, Bilecik Şeyh Edebali University, Bilecik 11230, Turkey; \\ ogokmen.yildiz@bilecik.edu.tr; Tel.: +90-228-214-1825
}

Received: 7 November 2018; Accepted: 24 December 2018; Published: 28 December 2018

check for updates

\begin{abstract}
In this paper, we construct a helicoidal surface with a prescribed weighted mean curvature and weighted extrinsic curvature in a 3-dimensional complete manifold with a positive density function. We get a result for the minimal case. Additionally, we give examples of a helicoidal surface with a weighted mean curvature and weighted extrinsic curvature.
\end{abstract}

Keywords: complete manifold; manifold with density; weighted curvature; helicoidal surface

\section{Introduction}

It is well known that a helicoidal surface is a generalization of a rotation surface. There are many studies about these surfaces under some given certain conditions [1-12]. Recently, the popular question has become whether a helicoidal surface can be constructed when its curvatures are prescribed. Several researchers have worked on this problem and obtained useful results. Firstly, Baikoussis et al. studied helicoidal surfaces with a prescribed mean and Gaussian curvature in $\mathbb{R}^{3}$ [13]. Then, Beneki et al. [14] and Ji et al. [15] studied similar work in $\mathbb{R}_{1}^{3}$. Furthermore, Dae Won Yoon et al. studied the helicoidal surfaces with a prescribed weighted mean and Gaussian curvature in $\mathbb{R}^{3}$ with density [16] and Ylldiz et al. have studied the helicoidal surfaces with prescribed weighted curvatures in $\mathbb{R}_{1}^{3}$ with density [17]. For more details on manifolds with density and surfaces in manifold with density, see References [18-25].

This problem is extended to complete manifolds. Lee et al. studied the helicoidal surfaces with a prescribed extrinsic curvature or mean curvature in a conformally flat 3-space [10]. It is well known that a metric on a complete manifold is conformal to the Euclidean metric. For a given surface in a complete manifold with a conformal factor function $F$, the mean curvature and the extrinsic curvature are given by:

$$
\begin{aligned}
H_{g_{F}} & =F H_{g_{0}}-\langle\mathbf{N}, \operatorname{grad} F\rangle, \\
G_{g_{F}} & =F^{2} G_{g_{0}}-2 H_{g_{0}} F\langle\mathbf{N}, \nabla F\rangle+\langle\mathbf{N}, \nabla F\rangle^{2},
\end{aligned}
$$

where $\mathbf{N}$ is the unit normal vector of a surface and $\nabla F$ is the gradient of $F, H_{g_{0}}$ is the mean curvature of the surface in Euclidean 3-space, and $G_{g_{0}}$ is the Gaussian curvature of a surface in Euclidean 3-space [26].

In this paper, we study helicoidal surfaces in a 3-dimensional complete manifold with density. We construct a helicoidal surface with a prescribed weighted mean and weighted extrinsic curvature. Then, we give examples to illustrate our result. 


\section{Preliminaries}

Let $M$ be a 3-dimensional complete manifold $\left(\mathbb{R}^{3},\langle,\rangle_{g}\right)$ equipped with a metric $\langle,\rangle_{g}$ that is conformal to the Euclidean metric $\langle$,$\rangle such that:$

$$
\langle,\rangle_{g}=\frac{1}{F^{2}}\langle,\rangle
$$

where $F: \mathbb{R}^{3} \rightarrow \mathbb{R}^{+}$is a positive differentiable function.

A manifold with a positive density function $\varphi$ is used to weight the volume and the hypersurface area. In terms of the underlying Riemannian volume $d V_{0}$ and area $d A_{0}$, the new, weighted volume and area are given by $d V=\varphi d V_{0}$ and $d A=\varphi d A_{0}$, respectively. One of the most important examples of manifolds with density, with applications to probability and statistics, is a Gauss space with density $\varphi=e^{a\left(-x^{2}-y^{2}-z^{2}\right)}$ for $a \in \mathbb{R}[22]$.

In Euclidean 3-space with density $e^{\varphi}$, the weighted mean curvature is given by:

$$
H_{\varphi_{g_{0}}}=H_{g_{0}}-\frac{1}{2}\langle\mathbf{N}, \nabla \varphi\rangle
$$

where $H_{g_{0}}$ is the mean curvature of the surface, $\mathbf{N}$ is the unit normal vector of the surface, and $\nabla \varphi$ is the gradient vector of $\varphi$ [23]. If $H_{\varphi_{g_{0}}}=0$, then the surface is called a weighted minimal surface. In Euclidean 3-space with density $e^{\varphi}$, the weighted Gaussian curvature with density is:

$$
G_{\varphi_{g_{0}}}=G_{g_{0}}-\triangle \varphi
$$

where $G_{g_{0}}$ is the Gaussian curvature of the surface and $\triangle$ is the Laplacian operator [27].

Throughout this paper, for $x=\left(x_{1}, x_{2}, x_{3}\right) \in \mathbb{R}^{3}$, we consider the positive density function and the conformal factor function as $e^{\varphi}=e^{-x_{1}^{2}-x_{2}^{2}}$ and $F=\sqrt{x_{1}^{2}+x_{2}^{2}}$, respectively.

Let $\gamma$ be a $C^{2}$-curve on $x_{1} x_{3}$-plane, of type $\gamma(u)=(u, 0, f(u))$, where $u \in I$ for an open interval $I \subset \mathbb{R}^{+}$. Using helicoidal motion on $\gamma$, we can obtain the helicoidal surface $M$ as:

$$
X(u, v)=\left[\begin{array}{ccc}
\cos v & -\sin v & 0 \\
\sin v & \cos v & 0 \\
0 & 0 & 1
\end{array}\right]\left[\begin{array}{c}
u \\
0 \\
f(u)
\end{array}\right]+\left[\begin{array}{c}
0 \\
0 \\
h v
\end{array}\right]
$$

with $x_{3}$-axis and a pitch $h \in \mathbb{R}$, so the parametric equation can be given in the form:

$$
X(u, v)=(u \cos v, u \sin v, f(u)+h v) .
$$

It is straightforward to see that the mean curvature $H_{g_{0}}$, the Gaussian curvature $G_{g_{0}}$, and the unit normal vector of helicoidal surface are:

$$
\begin{aligned}
H_{g_{0}} & =\frac{\left(u^{2}+h^{2}\right) u f^{\prime \prime}(u)+u^{2} f^{\prime 3}(u)+\left(u^{2}+2 h^{2}\right) f^{\prime}(u)}{2\left(u^{2} f^{\prime 2}(u)+u^{2}+h^{2}\right)^{3 / 2}}, \\
G_{g_{0}} & =\frac{u^{3} f^{\prime}(u) f^{\prime \prime}(u)-h^{2}}{\left(u^{2} f^{\prime 2}(u)+u^{2}+h^{2}\right)^{2}}, \\
\mathbf{N} & =\frac{\left(h \sin v-u f^{\prime}(u) \cos v,-u f^{\prime}(u) \sin v-h \cos v, u\right)}{\left(u^{2} f^{\prime 2}(u)+u^{2}+h^{2}\right)^{1 / 2}} .
\end{aligned}
$$


Using Equations (3) and (4), the weighted mean curvature $H_{\varphi_{g_{0}}}$ and the weighted Gaussian curvature $G_{\varphi_{g_{0}}}$ are obtained as:

$$
\begin{aligned}
H_{\varphi_{g_{0}}} & =\frac{\left(u^{2}+h^{2}\right) u f^{\prime \prime}(u)+\left(u^{2}-2 u^{4}\right) f^{\prime 3}(u)+\left(u^{2}+2 h^{2}-2 u^{4}-2 h^{2} u^{2}\right) f^{\prime}(u)}{2\left(u^{2} f^{\prime 2}(u)+u^{2}+h^{2}\right)^{3 / 2}}, \\
G_{\varphi_{g_{0}}} & =\frac{u^{3} f^{\prime}(u) f^{\prime \prime}(u)-h^{2}}{\left(u^{2} f^{\prime 2}(u)+u^{2}+h^{2}\right)^{2}}+4 .
\end{aligned}
$$

We assume that $M$ is a surface in a 3-dimensional complete manifold with density. By considering Equations (1)-(4), we can define the weighted mean curvature $H_{\varphi_{g_{F}}}$ and the weighted extrinsic curvature $G_{\varphi_{g_{F}}}$ as:

$$
\begin{aligned}
& H_{\varphi_{g_{F}}}=F H_{g_{0}}-\frac{1}{2} F\langle\mathbf{N}, \nabla \varphi\rangle-\langle\mathbf{N}, \nabla F\rangle \\
& G_{\varphi_{g_{F}}}=F^{2} G_{g_{0}}-2 F H_{g_{0}}\langle\mathbf{N}, \nabla F\rangle-F^{2} \triangle \varphi+\langle\mathbf{N}, \nabla \varphi\rangle\langle\mathbf{N}, \nabla F\rangle F+\langle\mathbf{N}, \nabla F\rangle^{2} .
\end{aligned}
$$

We obtain $H_{\varphi_{g_{F}}}$ and $G_{\varphi_{g_{F}}}$ for $M$ as:

$$
\begin{aligned}
H_{\varphi_{\text {SF }}}= & \frac{u\left[\left(3 u^{2}-2 u^{4}-2 h^{2}\left(u^{2}-2\right)\right) f^{\prime}(u)+\left(3 u^{2}-2 u^{4}\right) f^{\prime 3}(u)+u\left(u^{2}+h^{2}\right) f^{\prime \prime}(u)\right]}{2\left(u^{2} f^{\prime 2}(u)+u^{2}+h^{2}\right)^{3 / 2}}, \\
G_{\varphi_{\text {SF }}} & =\frac{\begin{array}{c}
u^{2}\left[4 h^{4}+4 u^{4}+h^{2}\left(8 u^{2}-1\right)+\left(h^{2}\left(6 u^{2}+3\right)+2\left(u^{2}+3 u^{4}\right)\right) f^{\prime^{2}}(u)\right. \\
\left.+2\left(u^{2}+u^{4}\right) f^{\prime 4}(u)+u\left(2 u^{2}+h^{2}\right) f^{\prime}(u) f^{\prime \prime}(u)\right]
\end{array}}{\left(u^{2} f^{\prime 2}(u)+u^{2}+h^{2}\right)^{2}} .
\end{aligned}
$$

\section{Helicoidal Surfaces with Prescribed Weighted Mean or Weighted Extrinsic Curvature}

In this section, we construct helicoidal surfaces with a prescribed weighted mean curvature and weighted extrinsic curvature in a 3-dimensional complete manifold with density $e^{\varphi}=e^{-x_{1}^{2}-x_{2}^{2}}$, where conformal factor $F=\sqrt{x_{1}^{2}+x_{2}^{2}}$ and $x=\left(x_{1}, x_{2}, x_{3}\right) \in \mathbb{R}^{3}$.

Theorem 1. Let $\gamma(u)=(u, 0, f(u))$ be a profile curve of the helicoidal surface given by $X(u, v)=$ $(u \cos v, u \sin v, f(u)+h v)$ in the 3-dimensional complete manifold with density and $H_{\varphi_{B_{F}}}(u)$ be the weighted mean curvature at the point $(u, 0, f(u))$. Then, there exists a two-parameter family of the helicoidal surface given by the curves:

$$
\gamma\left(u, H_{\varphi_{Q_{F}}}(u), h, c_{1}, c_{2}\right)=(u, 0, f(u)),
$$

where:

$$
f= \pm \int \frac{\sqrt{u^{2}+h^{2}}\left(\frac{1}{e^{-u^{2}} u^{4}}\left(\int\left(e^{-u^{2}} u^{4}\right) H_{\varphi_{g_{F}}} d u+c_{1}\right)\right)}{\sqrt{1-u^{2}\left(\frac{1}{e^{-u^{2}} u^{4}}\left(\int\left(e^{-u^{2}} u^{4}\right) H_{\varphi_{S_{F}}} d u+c_{1}\right)\right)^{2}}} d u+c_{2} .
$$

Conversely, for a given smooth function $H_{\varphi_{S_{F}}}(u)$, one can obtain the two-parameter family of curves $\gamma\left(u, H_{\varphi_{S_{F}}}(u), h, c_{1}, c_{2}\right)$ being the two-parameter family of helicoidal surfaces, accepting $H_{\varphi_{S_{F}}}(u)$ as the weighted mean curvature $h$ as a pitch.

Proof. Let us solve Equation (5), which is a second-order nonlinear ordinary differential equation. If we apply:

$$
\Psi=\frac{f^{\prime}(u)}{\sqrt{u^{2} f^{\prime 2}(u)+u^{2}+h^{2}}},
$$


into the equation, then we obtain the first-order linear ordinary differential equation:

$$
H_{\varphi_{g_{F}}}=\left(2 u-u^{3}\right) \Psi+\frac{u^{2}}{2} \Psi^{\prime}
$$

Then, the general solution of Equation (8) is:

$$
\Psi=\frac{1}{e^{-u^{2}} u^{4}}\left(\int\left(e^{-u^{2}} u^{4}\right) H_{\varphi_{g_{F}}} d u+c_{1}\right)
$$

where $c_{1} \in \mathbb{R}$. Using Equations (7) and (9), we obtain:

$$
\begin{aligned}
& \left(1-u^{2}\left(\frac{1}{e^{-u^{2}} u^{4}}\left(\int\left(e^{-u^{2}} u^{4}\right) H_{\varphi_{g_{F}}} d u+c_{1}\right)\right)^{2}\right) f^{\prime^{2}} \\
& =\left(u^{2}+h^{2}\right)\left(\frac{1}{e^{-u^{2}} u^{4}}\left(\int\left(e^{-u^{2}} u^{4}\right) H_{\varphi_{g_{F}}} d u+c_{1}\right)\right)^{2} .
\end{aligned}
$$

From the above equation, we obtain:

$$
f^{\prime}= \pm \frac{\sqrt{u^{2}+h^{2}} \Psi}{\sqrt{1-u^{2} \Psi^{2}}}
$$

By integrating Equation (11), we obtain:

$$
f= \pm \int \frac{\sqrt{u^{2}+h^{2}} \Psi}{\sqrt{1-u^{2} \Psi^{2}}} d u+c_{2}
$$

where $c_{2} \in \mathbb{R}$.

By contrast, for a given constant $h \in \mathbb{R}-\{0\}$, a real-valued smooth function $H_{\varphi_{g_{F}}}(u)$ defined on an open interval $I \subset \mathbb{R}^{+}$and an arbitrary $u_{0} \in I$, there exists an open subinterval $u_{0} \in I^{\prime} \subset I$ and an open interval $J \subset \mathbb{R}$ which contains:

$$
\widetilde{c}_{1}=-\left(\int\left(e^{-u^{2}} u^{4}\right) H_{\varphi_{g_{F}}} d u\right)\left(u_{0}\right),
$$

such that:

$$
S\left(u, c_{1}\right)=1-u^{2}\left(\frac{1}{e^{-u^{2}} u^{4}}\left(\int\left(e^{-u^{2}} u^{4}\right) H_{\varphi_{g_{F}}} d u+c_{1}\right)\right)^{2}>0,
$$

for arbitrary $\left(u, c_{1}\right)$. Since $S\left(u_{0}, \widetilde{c}_{1}\right)=1>0$ and $S$ is continuous, $S$ is positive on $I^{\prime} \times J \subset \mathbb{R}^{2}$. Thus, the two-parameter family of the curves can be given as:

$$
\gamma\left(u, H_{\varphi_{g_{F}}}(u), h, c_{1}, c_{2}\right)=(u, 0, f(u))
$$

where:

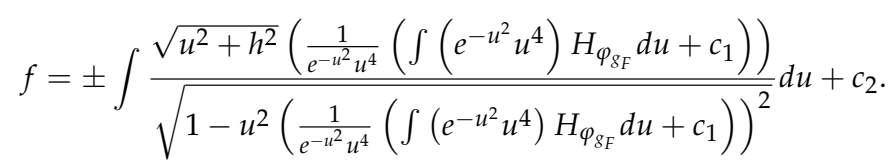

The following corollary is an immediate consequence of Theorem 1 and the definition of a minimal surface.

Corollary 1. Let $M$ be a minimal helicoidal surface in a complete manifold with density $e^{\varphi .}$ Then, $M$ is an open part of either a helicoid or a surface parametrized by:

$$
X(u, v)=\left(u \cos v, u \sin v, \pm \int \frac{c_{1} \sqrt{u^{2}+h^{2}}}{\sqrt{e^{-2 u^{2} u^{8}-u^{2} c_{1}^{2}}}} d u+c_{2}+h v\right)
$$

where $c_{1}, c_{2} \in \mathbb{R}$. 
Example 1. Consider a helicoidal surface with the weighted mean curvature:

$$
H_{\varphi_{g_{F}}}=\frac{5 e^{-u^{2}} u^{4}-2 e^{-u^{2}} u^{6}}{e^{-u^{2}} u^{4}},
$$

and the pitch $h=1$ in a complete manifold with density. Using Equation (12), we get $\gamma(u)$. Thus, we obtain the parametrization of the surface as follows:

$$
X(u, v)=\left(u \cos v, u \sin v,-\sqrt{1-u^{2}}+v\right),
$$

and the figure of the domain:

$$
\left\{\begin{array}{c}
0<u<1 \\
-4<v<4
\end{array},\right.
$$

is given in Figure 1.

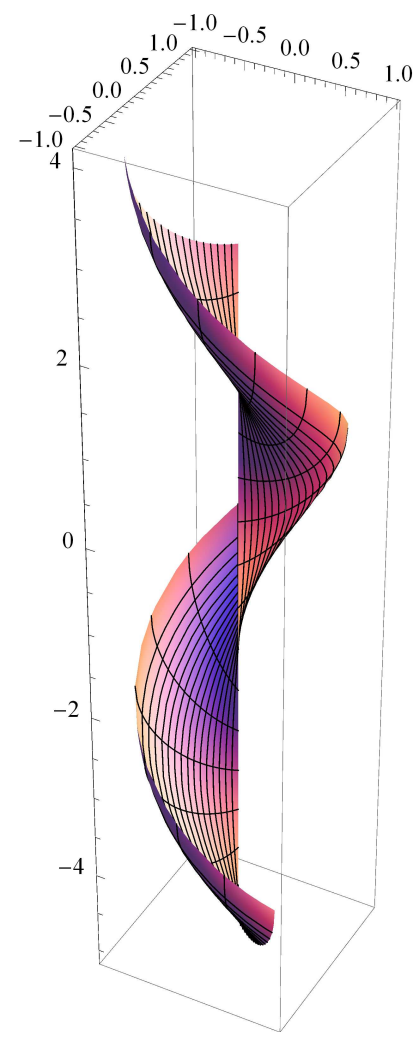

Figure 1. The helicoidal surface with the weighted mean curvature.

The difference between $H_{\varphi_{g_{0}}}$ and $H_{\varphi_{g_{F}}}$ of the helicoidal surface with density can be seen in Figure 2.

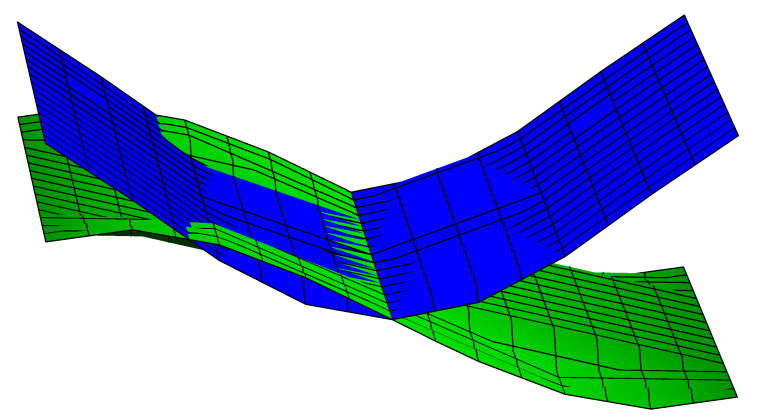

Figure 2. $H_{\varphi_{g_{0}}}$ (Green) and $H_{\varphi_{g_{F}}}$ (Blue). 
Example 2. Consider a helicoidal surface with the weighted mean curvature:

$$
H_{\varphi_{g_{F}}}=\frac{1-2 u^{2}}{u^{4}}
$$

and the pitch $h=1$ in a complete manifold with density. Using Equation (12), we get $\gamma(u)$. Thus, we obtain the parametrization of the surface as follows:

$$
X(u, v)=\left(u \cos v, u \sin v,-\frac{\sqrt{-1+u^{4}} \arctan \left(\frac{\sqrt{1+u^{2}}}{\sqrt{-1+u^{4}}}\right)}{\sqrt{1-\frac{1}{u^{4}}} u^{2}}+v\right),
$$

and the figure of the domain:

$$
\left\{\begin{array}{c}
2<u<10 \\
-5<v<5
\end{array}\right.
$$

is given in Figure 3.

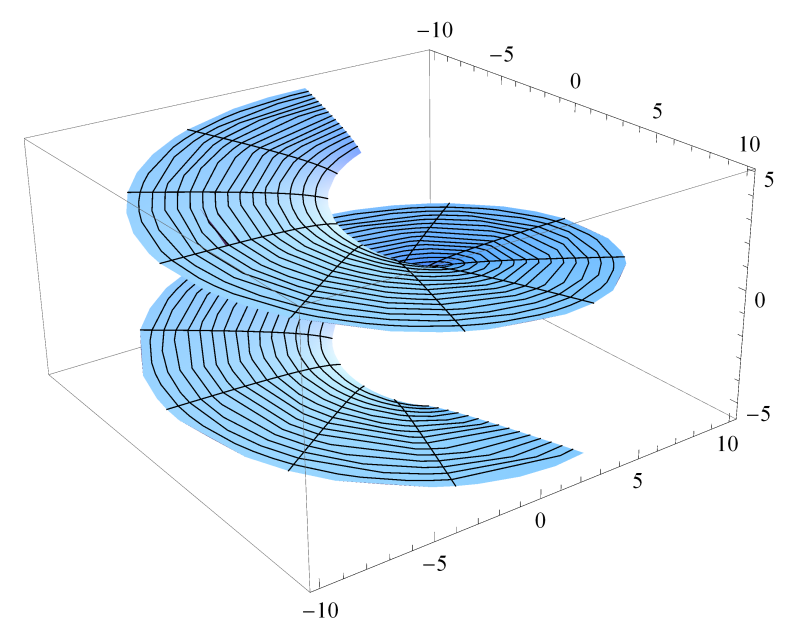

Figure 3. The helicoidal surface with the weighted mean curvature.

Theorem 2. Let $\gamma(u)=(u, 0, f(u))$ be a profile curve of the helicoidal surface given by $X(u, v)=$ $(u \cos v, u \sin v, f(u)+h v)$ in a 3-dimensional complete manifold with density and $G_{\varphi_{g_{F}}}(u)$ be the weighted extrinsic curvature at the point $(u, 0, f(u))$. Then, there exists a two-parameter family of the helicoidal surface, which is given by the curves:

$$
\gamma\left(u, G_{\varphi_{g_{F}}}, h, c_{1}, c_{2}\right)=\left(u, 0, \pm \int\left(\frac{\left(e^{-u^{2}} u^{5}\left(h^{2}+2 u^{2}\right)^{-1-\frac{h^{2}}{2}}\right) u+\left(u^{2}+h^{2}\right) B}{\left(e^{-u^{2} u^{5}\left(h^{2}+2 u^{2}\right)^{-1-\frac{h^{2}}{2}}}\right) u-u^{2} B}\right)^{\frac{1}{2}} d u+c_{2}\right)
$$

where:

$$
B=\int\left(e^{-u^{2}} u^{5}\left(h^{2}+2 u^{2}\right)^{-1-\frac{h^{2}}{2}}\right)\left(\left(\frac{-4-8 h^{2}-12 u^{2}}{2 u^{2}+h^{2}}\right)+\frac{2}{u^{2}} G_{\varphi_{S_{F}}}\right) d u+c_{1},
$$

and $c_{1}$ and $c_{2}$ are constants. Conversely, for a given smooth function $G_{\varphi_{g_{F}}}$, one can obtain the two-parameter family of curves $\gamma\left(u, G_{\varphi_{g_{F}}}(u), h, c_{1}, c_{2}\right)$, being the two-parameter family of the helicoidal surfaces, accepting $G_{\varphi_{g_{F}}}$ as the weighted extrinsic curvature $h$ as a pitch.

Proof. Let's solve the second-order nonlinear ordinary differantial Equation (6). We can rewrite Equation (6) as follows:

$$
\Phi^{\prime}+\left(\frac{5 h^{2}+6 u^{2}-4 h^{2} u^{2}-4 u^{4}}{2 u^{3}+u h^{2}}\right) \Phi=\left(\frac{-4-8 h^{2}-12 u^{2}}{2 u^{2}+h^{2}}\right)+\frac{2}{u^{2}} G_{\varphi_{g_{F}}}
$$

where:

$$
\Phi=\frac{u f^{\prime 2}(u)-u}{u^{2} f^{\prime 2}(u)+u^{2}+h^{2}}
$$


The general solution of Equation (13) is:

$$
\Phi=\frac{\left(\int\left(e^{-u^{2}} u^{5}\left(h^{2}+2 u^{2}\right)^{-1-\frac{h^{2}}{2}}\right)\left(\left(\frac{-4-8 h^{2}-12 u^{2}}{2 u^{2}+h^{2}}\right)+\frac{2}{u^{2}} G_{\varphi_{S_{F}}}\right) d u+c_{1}\right)}{e^{-u^{2}} u^{5}\left(h^{2}+2 u^{2}\right)^{-1-\frac{h^{2}}{2}}},
$$

where $c_{1} \in \mathbb{R}$. Combining Equations (14) and (15),we get:

$$
\begin{aligned}
& \left(\left(e^{-u^{2}} u^{5}\left(h^{2}+2 u^{2}\right)^{-1-\frac{h^{2}}{2}}\right) u\right. \\
& \left.-u^{2}\left(\int\left(e^{-u^{2}} u^{5}\left(h^{2}+2 u^{2}\right)^{-1-\frac{h^{2}}{2}}\right)\left(\left(\frac{-4-8 h^{2}-12 u^{2}}{2 u^{2}+h^{2}}\right)+\frac{2}{u^{2}} G_{\varphi_{S_{F}}}\right) d u+c_{1}\right)\right) f^{\prime 2}(u) \\
& =u\left(e^{-u^{2}} u^{5}\left(h^{2}+2 u^{2}\right)^{-1-\frac{h^{2}}{2}}\right) \\
& +\left(u^{2}+h^{2}\right)\left(\int\left(e^{-u^{2}} u^{5}\left(h^{2}+2 u^{2}\right)^{-1-\frac{h^{2}}{2}}\right)\left(\left(\frac{-4-8 h^{2}-12 u^{2}}{2 u^{2}+h^{2}}\right)+\frac{2}{u^{2}} G_{\varphi_{S_{F}}}\right) d u+c_{1}\right) .
\end{aligned}
$$

If we set:

$$
B=\int\left(e^{-u^{2}} u^{5}\left(h^{2}+2 u^{2}\right)^{-1-\frac{h^{2}}{2}}\right)\left(\left(\frac{-4-8 h^{2}-12 u^{2}}{2 u^{2}+h^{2}}\right)+\frac{2}{u^{2}} G_{\varphi_{g_{F}}}\right) d u+c_{1}
$$

then:

$$
f^{\prime^{2}}=\frac{\left(e^{-u^{2}} u^{5}\left(h^{2}+2 u^{2}\right)^{-1-\frac{h^{2}}{2}}\right) u+\left(u^{2}+h^{2}\right) B}{\left(e^{-u^{2} u^{5}\left(h^{2}+2 u^{2}\right)^{-1-\frac{h^{2}}{2}}}\right) u-u^{2} B} .
$$

It follows that:

$$
f(u)= \pm \int\left(\frac{\left(e^{-u^{2}} u^{5}\left(h^{2}+2 u^{2}\right)^{-1-\frac{h^{2}}{2}}\right) u+\left(u^{2}+h^{2}\right) B}{\left(e^{-u^{2}} u^{5}\left(h^{2}+2 u^{2}\right)^{-1-\frac{h^{2}}{2}}\right) u-u^{2} B}\right)^{\frac{1}{2}} d u+c_{2}
$$

where $c_{2} \in \mathbb{R}$.

Conversely, for a given $h \in \mathbb{R}$ and a smooth function $G_{\varphi_{g_{F}}}(u)$, defined on an open interval $I \subset \mathbb{R}^{+}$and an arbitrary $u_{0} \in I$, there exists an open subinterval $I^{\prime} \subset I$ containing $u_{0}$ and an open interval $J \subset \mathbb{R}$ containing:

$$
\widetilde{c}_{1}=-\left(\int\left(e^{-u^{2}} u^{5}\left(h^{2}+2 u^{2}\right)^{-1-\frac{h^{2}}{2}}\right)\left(\left(\frac{-4-8 h^{2}-12 u^{2}}{2 u^{2}+h^{2}}\right)+\frac{2}{u^{2}} G_{\varphi_{g F}}\right) d u\right)\left(u_{0}\right)
$$

such that:

$$
S\left(u, c_{1}\right)=\left(e^{-u^{2}} u^{5}\left(h^{2}+2 u^{2}\right)^{-1-\frac{h^{2}}{2}}\right)-u B>0,
$$

which is defined on $I^{\prime} \times J$. Thus, a two-parameter family of the curves can be given as:

$$
\gamma\left(u, G_{\varphi_{g_{F}}}, h, c_{1}, c_{2}\right)=\left(u, 0, \pm \int\left(\frac{\left(e^{-u^{2}} u^{5}\left(h^{2}+2 u^{2}\right)^{-1-\frac{h^{2}}{2}}\right) u+\left(u^{2}+h^{2}\right) B}{\left(e^{-u^{2}} u^{5}\left(h^{2}+2 u^{2}\right)^{-1-\frac{h^{2}}{2}}\right) u-u^{2} B}\right)^{\frac{1}{2}} d u+c_{2}\right)
$$

where $\left(u, c_{1}\right) \in I^{\prime} \times J ; c_{2}, h \in \mathbb{R}$ and $G_{\varphi_{g_{F}}}$ is a smooth function.

Example 3. Consider a helicoidal surface with the weighted extrinsic curvature:

$$
G_{\varphi_{g_{F}}}(u)=\frac{4+11 u^{2}+14 u^{4}+4 u^{6}}{\left(2+u^{2}\right)^{2}}
$$


in a complete manifold with density. Using Equation (17), we obtain $f(u)=\ln u$ for $h=1, c_{1}=0, c_{2}=0$ and the parametrization of the surface as follows:

$$
X(u, v)=(u \cos v, u \sin v, \ln u+v) .
$$

The figure of the surface of the domain:

$$
\left\{\begin{array}{c}
0<u<3 \\
-4<v<4
\end{array},\right.
$$

is given in Figure 4.

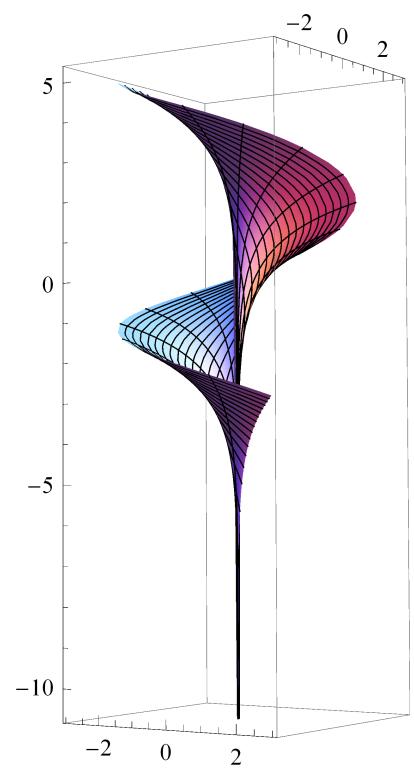

Figure 4. The helicoidal surface with the weighted Gaussian curvature.

The difference between $G_{\varphi_{g_{0}}}$ and $G_{\varphi_{g_{F}}}$ of the helicoidal surface with density can be seen in Figure 5.

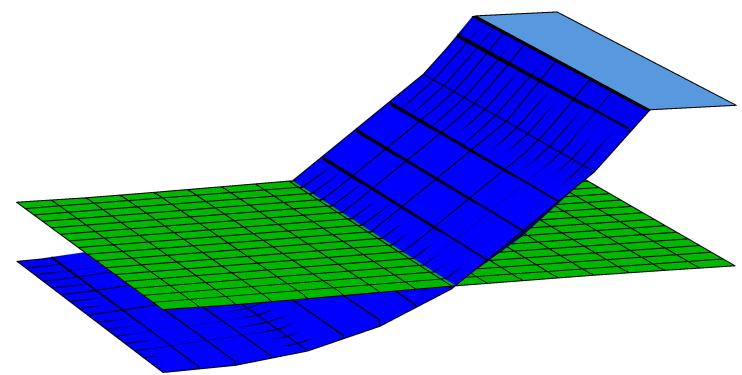

Figure 5. $G_{\varphi_{g_{0}}}$ (Green) and $G_{\varphi_{g_{F}}}$ (Blue).

\section{Conclusions and Future Work}

In this paper, using the conformal factor function $F=\sqrt{x_{1}^{2}+x_{2}^{2}}$, we constructed a helicoidal surface with a prescribed weighted mean curvature and Gaussian curvature in a complete manifold with a positive density function. Different helicoidal surfaces can be obtained in a complete manifold with density using different conformal factor functions. In addition, if conformal factor function $F$ is bounded, a manifold is called a conformally flat space. Thus, by considering a bounded function, one can study helicoidal surface in a conformally flat space with density.

Funding: This research received no external funding.

Conflicts of Interest: The author declares no conflict of interest. 


\section{References}

1. Araujo, K.O.; Cui, N.; Pina, R.D.S. Helicoidal minimal surfaces in a conformally flat 3-space. Bull. Korean Math. Soc. 2016, 53, 531-540. [CrossRef]

2. Baba-Hamed, C.; Bekkar, M. Helicoidal surfaces in the three-dimensional Lorentz-Minkowski space satisfying $\triangle^{I I} r_{i}=\lambda_{i} r_{i}$.J. Geom. 2011, 100,1. [CrossRef]

3. Choi, M.K.; Kim, Y.H.; Liu, H.; Yoon, D.W. Helicoidal surfaces and their Gauss map in Minkowski 3-space. Bull. Korean Math. Soc. 2010, 47, 859-881. [CrossRef]

4. Delaunay, C.H. Sur la surface de révolution dont la courbure moyenne est constante. J. Math. Appl. 1841, 6, 309-314.

5. Do Carmo, M.P.; Dajczer, M. Helicoidal surfaces with constant mean curvature. Tohoku Math. J. 1982, 34, 425-435.

6. Güler, E.; Turgut Vanli, A. On the mean, gauss, the second gaussian and the second mean curvature of the Helicoidal surfaces with light-like axis in $\mathbb{R}_{1}^{3}$. Tsukuba J. Math. 2008, 32, 49-65. [CrossRef]

7. Güler, E. A new kind helicoidal surface of value m. Int. Electron. J. Geom. 2014, 7, 154-162.

8. Güler, E. Isometric deformation of $(m, n)$-type helicoidal surface in the three dimensional Euclidean Space. Mathematics 2018, 6, 226. [CrossRef]

9. Ji, F.; Hou, Z.H. Helicoidal surfaces under the cubic screw motion in Minkowski 3-space. J. Math. Anal. Appl. 2006, 318, 634-647. [CrossRef]

10. Lee, C.W.; Lee, J.W.; Yoon, D.W. On Helicoidal Surfaces in a Conformally Flat 3-Space. Mediterr. J. Math. 2017, 14, 164. [CrossRef]

11. Rafael, L.; Demir, E. Helicoidal surfaces in Minkowski space with constant mean curvature and constant Gauss curvature. Open Math. 2014, 12, 1349-1361.

12. Roussos, I.M. The helicoidal surfaces as Bonnet surfaces. Tohoku Math. J. Second Ser. 1988, 40, 485-490. [CrossRef]

13. Baikoussis, C.; Koufogiorgos, T. Helicoidal surfaces with prescribed mean or Gaussian curvature. J. Geom. 1998, 63, 25-29. [CrossRef]

14. Beneki C.C.; Kaimakamis, G.; Papantoniou, B.J. Helicoidal surfaces in three-dimensional Minkowski space. J. Math. Anal. Appl. 2002, 275, 586-614. [CrossRef]

15. Ji, F.; Hou, Z.H. A kind of helicoidal surfaces in 3-dimensional Minkowski space. J. Math. Anal. Appl. 2005, 304, 632-643. [CrossRef]

16. Yoon, D.W.; Kim, D.S.; Kim, Y.H.; Lee, J.W. Constructions of Helicoidal Surfaces in Euclidean Space with Density. Symmetry 2017, 9, 173. [CrossRef]

17. Yıldız, Ö.G.; Hızal, S.; Akyiğit, M. Type $I^{+}$Helicoidal Surfaces with Prescribed Weighted Mean or Gaussian Curvature in Minkowski Space with Density. Anal. Univ. “Ovidius" Constanta-Ser. Mat. 2018, 26, 1-11.

18. Hieu, D.T.; Hoang, N.M. Ruled minimal surfaces in $\mathbb{R}^{3}$ with density $e^{z}$. Pac. J. Math. 2009, 243, $277-285$.

19. Morgan, F. Geometric Measure Theory: A Beginner's Guide; Academic Press: New York, NY, USA, 2016.

20. Morgan, F. Manifolds with density. Not. AMS 2005, 52, 853-858.

21. Morgan, F. Myers' theorem with density. Kodai Math. J. 2006, 29, 455-461. [CrossRef]

22. Morgan, F. Manifolds with Density and Perelman's Proof of the Poincaré Conjecture. Am. Math. Mon. 2009, 116, 134-142. [CrossRef]

23. Rayón, P.; Gromov, M. Isoperimetry of waists and concentration of maps. Geom. Funct. Anal. 2003, 13, $178-215$. [CrossRef]

24. Rosales, C.; Cañete, A.; Bayle, V.; Morgan, M. On the isoperimetric problem in Euclidean space with density. Calcul. Var. Partial Differ. Equ. 2008, 31, 27-46. [CrossRef]

25. Yoon, D.W. Weighted minimal translation surfaces in Minkowski 3-space with density. Int. J. Geom. Methods Mod. Phys. 2017, 14, 1750178. [CrossRef] 
26. Pina A.V.R.; Souza, M. Surfaces of rotation with constant extrinsic curvature in a conformally flat 3-space. Results Math. 2011, 60, 225.

27. Corwin, I.; Hoffman, N.; Hurder, S.; Šešum, V.; Xu, Y. Differential geometry of manifolds with density. Rose-Hulman Undergrad. Math. J. 2006, 7, 1-15. 